Research Article

\title{
A Network Pharmacology-Based Study on Irritable Bowel Syndrome Prevention and Treatment Utilizing Shenling Baizhu Powder
}

\author{
Meng Meng $\mathbb{D},{ }^{1}$ Chen Bai $\mathbb{D},{ }^{2}$ Bo Wan $\mathbb{D},,^{3}$ Luqing Zhao $\mathbb{D},{ }^{1}$ Zhe $\operatorname{Li}\left(\mathbb{D},{ }^{1}\right.$ Danyan Li $\mathbb{D},{ }^{1}$ \\ and Shengsheng Zhang $\mathbb{1}^{1}$ \\ ${ }^{1}$ Digestion Center, Beijing Hospital of Traditional Chinese Medicine, Capital Medical University, Beijing, China \\ ${ }^{2}$ Beijing University of Chinese Medicine, Beijing, China \\ ${ }^{3}$ Center for Stem Cells and Regenerative Medicine, King's College London, Guy's Hospital, Great Maze Pond, London, UK
}

Correspondence should be addressed to Shengsheng Zhang; zhangshengsheng@bjzhongyi.com

Received 29 April 2021; Accepted 3 November 2021; Published 23 November 2021

Academic Editor: Kazim Husain

Copyright (C) 2021 Meng Meng et al. This is an open access article distributed under the Creative Commons Attribution License, which permits unrestricted use, distribution, and reproduction in any medium, provided the original work is properly cited.

Background and Objective. Irritable bowel syndrome (IBS) is a prevalent disorder of the gastrointestinal system with complex pathogenesis. Shenling Baizhu powder (SLBZP) is a Chinese herbal compound with multicomponent and multitarget characteristics. Increasing volumes of evidence demonstrate that it has a notable therapeutic impact on IBS. This study therefore is aimed at exploring the potential effective components of SLBZP and their mechanisms in IBS treatment utilizing network pharmacology. Methods. Metabolomics was used to detect the secondary metabolites in SLBZP; the target protein was acquired by target fishing according to the compound's structure. The SymMap database was used to search herbal medicines for the target protein. The target gene of IBS gave rise to the common gene protein which is the potential target of SLBZP in IBS therapy. The interactions between target proteins were analyzed in a STRING database, the protein relationship network was analyzed using Cytoscape software, and the Kyoto Encyclopedia of Genes and Genomes enrichment analysis of the core target gene group was carried out in a DAVID database in order to construct the "compound-traditional Chinese medicine/ molecule-target-pathway" network. Molecular docking was used to verify the core protein and its related small molecular compounds. Result. There were 129 types of secondary metabolites in SLBZP. 80 target proteins of these metabolites were potential core targets for IBS treatment including acetylcholinesterase (AChE), arachidonate-5-lipoxygenase (ALOX5), B-cell lymphoma-2 (BCL2), recombinant cyclin D1 (CCND1), and catenin- $\beta 1$ (CTNNB1), among others. Results from these targets indicated that the most enriched pathway was the tumor necrosis factor (TNF) signaling pathway $(p<0.001)$ and that the most abundant pathway was signal transduction. In the network nodes of the TNF signaling pathway, the Chinese medicines with the highest aggregation were Lablab semen album and Glycyrrhizae radix et rhizoma (degree $=11)$. The small molecules

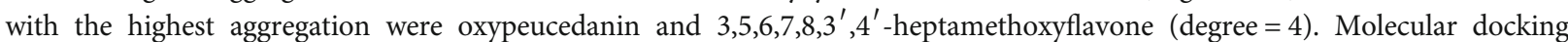
results confirmed that daidzein 7-O-glucoside (daidzin) had the highest degree of binding to TNF proteins in the TNF signaling pathway. Conclusion. This study shows that SLBZP can treat IBS by influencing multiple targets and pathways, of which the TNF signaling pathway may be the most significant. This typifies the pharmacological characteristics of traditional Chinese medicine, i.e., multiple targets, numerous pathways, and specific therapeutic effects on diseases. SLBZP can therefore be used as a candidate drug for clinical IBS by intervening in human signal transduction.

\section{Background}

Irritable bowel syndrome (IBS) is one of the most frequently arising pathologies of the gastrointestinal tract presenting in the clinic. Its main manifestations are pain and discomfort within the abdomen, together with changes in bowel habits. With an incidence rate range of $7 \%$ in Southeast Asia to $21 \%$ in South America [1], IBS affects hundreds of millions of people. The pathogenic factors include alterations in the microbiome, permeability, peristalsis, and immune function 
TABLE 1: Research overview of studies relating to SLBZP in the treatment of digestive system diseases, listed in descending order of publication year.

\begin{tabular}{lccc}
\hline Article & Disease & Research & Position of action mechanism \\
\hline Chao et al. [5] & Ulcerative colitis & Mice & The MAPK/NF- $\kappa$ B and pyroptosis signaling pathway \\
Ji et al. [6] & Chronic diarrhea & Rats & Intestinal absorption function and mucosal ultrastructure \\
Zhang et al. [7] & Functional dyspepsia & Rats & Intestinal microbiota \\
Lv et al. [8] & Inflammatory bowel disease & Rats & Intestinal microbiota \\
Shi et al. [9] & Diarrhea & Rats & Intestinal microbiota \\
Lv et al. [10] & Antibiotic-associated diarrhea & Rats & Intestinal microbiota \\
Son et al. [11] & Cancer of the liver & Mice & Tumor growth promoter, antiapoptotic protein, and proapoptotic \\
& Nonalcoholic fatty liver & Rat Kupffer & p38 MAPK pathway \\
Yang et al. [12] & disease & cells & Ghrelin \\
Chao et al. & Colon cancer & Human & \\
[13] & & &
\end{tabular}

of the intestine, as well as changes in the brain-gut axis and social psychological status, among others [2]. IBS is divided into four subtypes according to defecation habits: (i) constipation (IBS-C); (ii) diarrhea (IBS-D); (iii) mixed (IBS-M); and (iv) undetermined (IBS-U). The first-line treatment of IBS usually includes exercise and diet control, fiber supplements, probiotics, antispasmodics, and secretagogues [3]. Although IBS is a prevalent complaint and these drugs target specific symptoms, they have little effect on systemic symptomatology or abdominal pain and distension [4].

Shenling Baizhu powder (SLBZP) is derived from the "Prescriptions of Taiping Heji Bureau" (1148 A.D.) in the Song Dynasty of China. Its constituents include white lentils, Atractylodes macrocephala, Poria cocos, licorice, Platycodon grandiflorum, lotus seed, ginseng, Amomum villosum, yam, and coix seed. The main clinical effects of the drug are splenic and gastric fortification and replenishment and restoration of lung qi. In China, it is principally used to alleviate clinical symptoms such as splenic and gastric inadequacy, anorexia, loose motions, shortness of breath, cough, and limb fatigue. To date, several pharmacological studies have been published relating to SLBZP, as shown in Table 1, suggesting that it can exert therapeutic effects on several gastrointestinal diseases via a spectrum of pharmacological approaches [5-13]. A systematic review of studies evaluating the treatment of IBS with SLBZP indicated that the mixture was safe and effective in enhancing the cure rate of IBS and diminishing the severity of diarrhea, abdominal pain, and distension [14]. However, there is little research relating to its mechanism of action in such patients.

In 2007, Hopkins, from the United Kingdom, proposed the concept of network pharmacology which takes the biological databases as the processing object [15]. From the perspective of systems biology, using an empirical interaction network of pathologies, phenotypes, genomes, targets, and pharmaceutical agents, network pharmacology facilitates the observation of the intervention and influence of drugs on the pathological network. This is achieved through network topology, target criterion, and classification analyses, respectively, together with other computational analytical methods, enabling drug research and development protocols to resemble actual pathological scenarios more closely. Network pharmacology systematically understands the dynamic changes of human diseases and the interaction of multiple factors in the pathophysiological process. This is highly consistent with the characteristics of the numerous drug components and corresponding multiple targets of compounds utilized in traditional Chinese medicine.

A network pharmacology method was deployed in the current research in order to construct the "compoundtraditional Chinese medicine/molecule-target-pathway" network of SLBZP for the treatment of IBS and to explore its potential pharmacological mechanism. The flow chart of the entire research design is shown in Figure 1. The potential targets of SLBZP with respect to IBS therapy were acquired using metabolomics technology, target fishing, and database matching methods. The core targets were enriched and analyzed, and then the "compound-traditional Chinese medicine/molecule-target-pathway" network was configured. The results obtained during this work will offer a data reference for the research and development of de novo drugs for other treatable diseases and IBS with respect to SLBZP.

\section{Results}

2.1. Compound Secondary Metabolites. As shown in Figure 2, a total of 129 secondary metabolites were recognized within the compound granules, which were sorted in descending order according to the integrated area of mass to charge ratio mass spectrometry response. The top five percent were as follows: bis(N,N-diethylethanaminium)-2-acetamido-1,5-anhydro-2-deoxy-1-[-hydroxy(phosphonato)methyl]-D-glucitol (relative content $6.49 \times 10^{7}$ ); glycyroside (relative content $4.81 \times 10^{7}$ ); apigenin 6,8-C-diglucoside (relative content 4.20 $\times 10^{7}$ ); acacetin-7-O-glucuronide (relative content $4.00 \times$ $\left.10^{7}\right)$; and trigonelline (relative content $3.88 \times 10^{7}$ ). Fiftyeight types of flavonoids were noted, of which the following 21 were most abundant (as shown in Figure 3): apigenin 6,8C-diglucoside; acacetin-7-O-glucuronide; licoflavone C; acacetin-7-O-galactoside; luteolin-7-O-rutinoside; isosinensetin; 


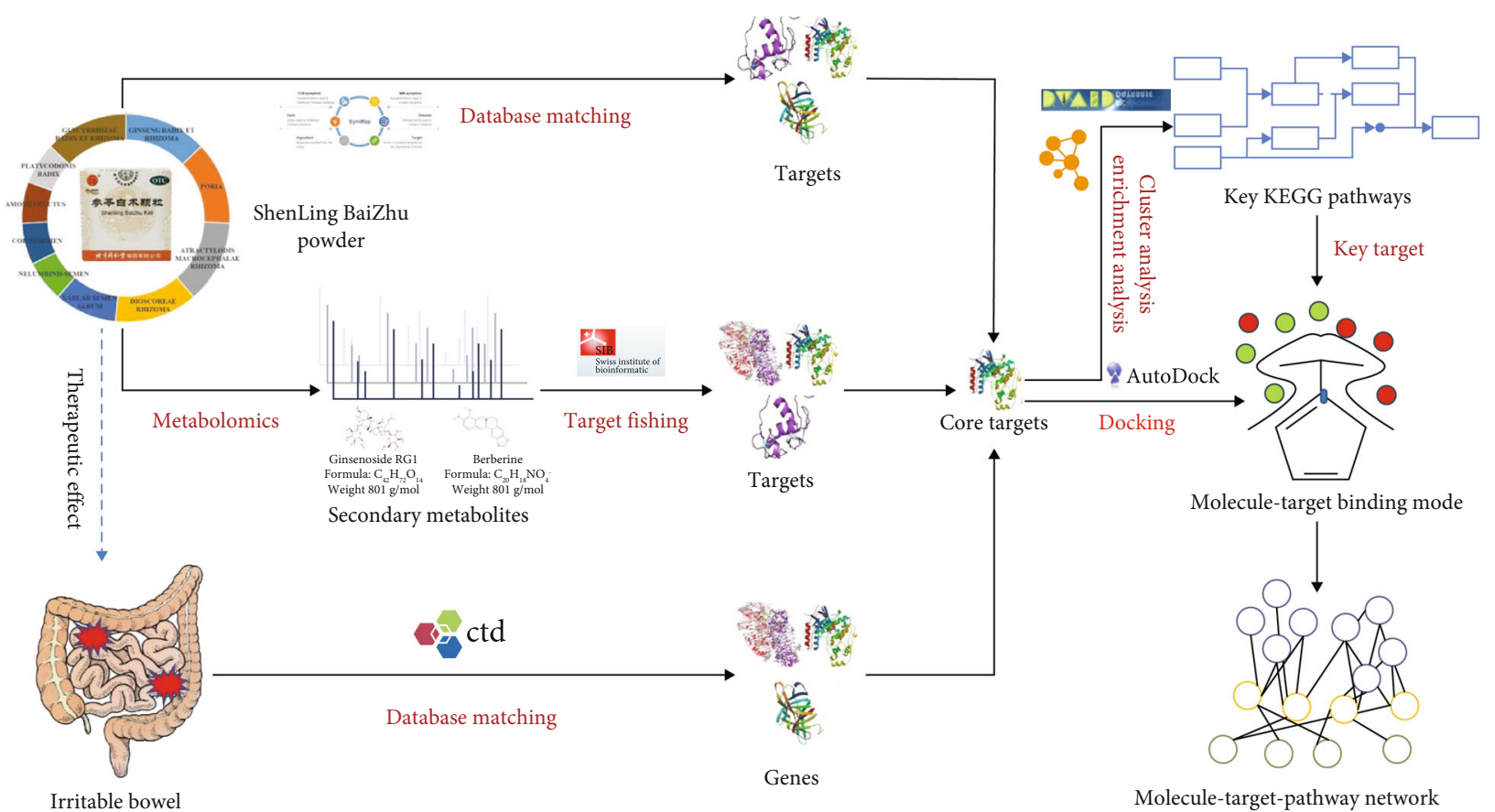

FIGURE 1: Research design flow chart.

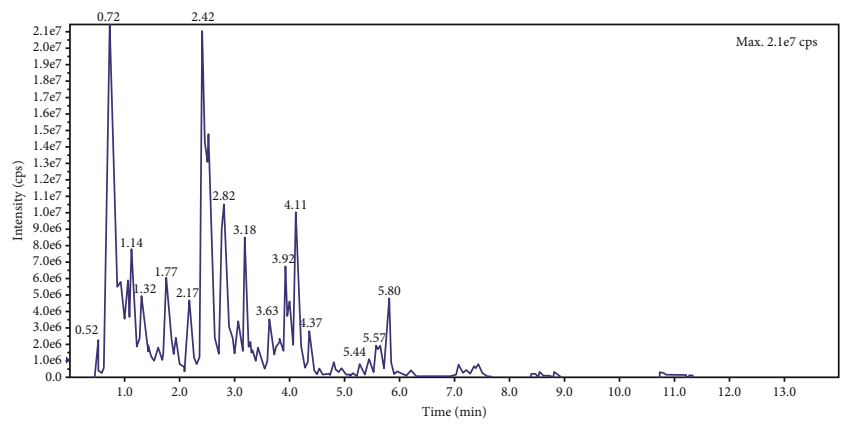

- TIC of -MRM (210 pair); from sample 1 (M)
20200304. wiff (turbo spray IonDrive)

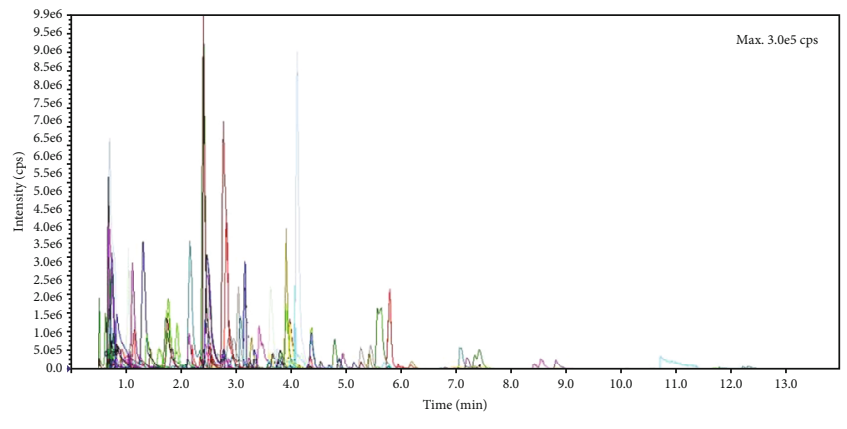

XIC of-MRM (210 pairs); 341.109/119.000 amu e
MWXS-19-701-2___WH $6500-5$ C C07_MWDB4...

(b)

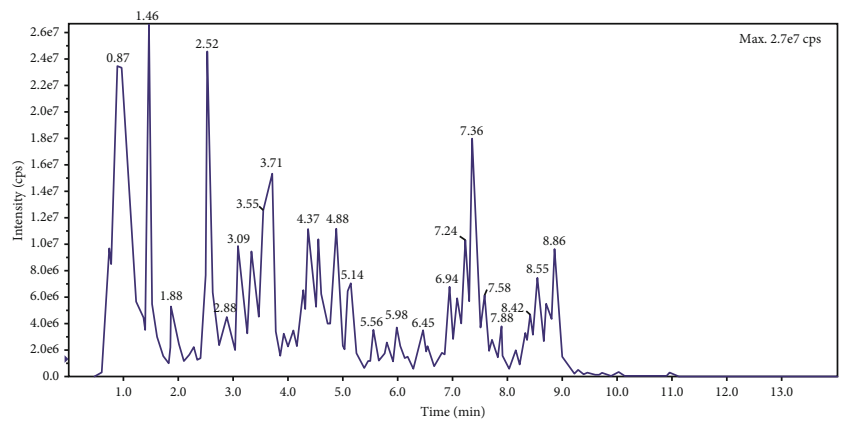

- TIC of +MRM (866 pairs); from sample 19 (MWXS19701-2_mix01_P) of MWXS-19-701-2___WHH6500-5_C07_MWDB4.0_ZX_
20200304. wiff (turbo spray IonDrive)

(a)

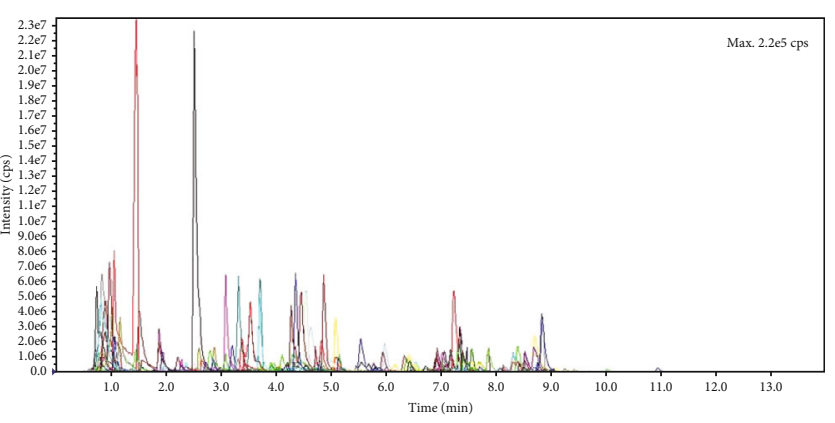

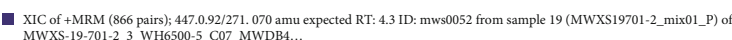

FIGURE 2: Qualitative and quantitative detection results of secondary metabolites in compound granules. Note that (a) shows the total ion flow diagram of mass spectrometry analysis of mixed samples and (b) shows the multipeak diagram of MRM metabolite detection. Left: positive ion current; right: negative ion current. 


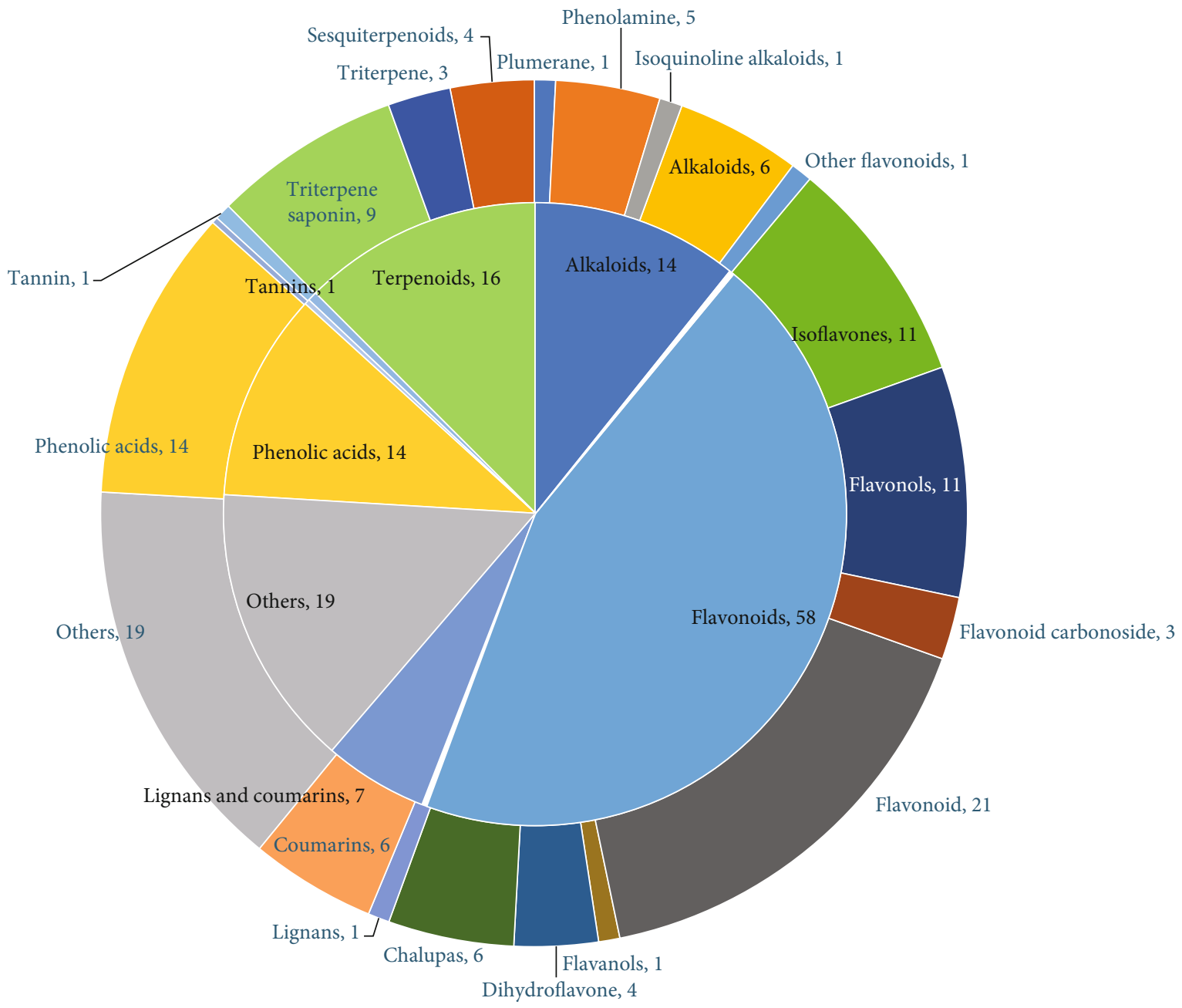

FIGURE 3: Primary and secondary classifications of secondary metabolites. Note that the number is the number of detection times. The inner and outer circles are the first- and second-level classifications, respectively.

luteolin-6,8-di-C-glucoside; diosmin; luteolin-7-O-glucoside (cynaroside); nobiletin; baicalin; luteolin-7-O-glucuronide; diosmetin-7-O-galactoside; 5-hydroxy-6,7,8,3', $4^{\prime}$-pentamethoxyflavone; 8-methoxychrysin (wogonin); tetramethylO-isoscutellarein; diosmetin-7-O-glucuronide; isorhamnetin3-O-rutinoside (Narcissin); Sudachiin B; apigenin; and apigenin O-hexosyl-O-pentoside.

2.2. Targets of SLBZP in the Treatment of IBS. As shown in Figure 4, a total of 1073 secondary metabolite-related targets, 618 targets included in the database, and 456 IBSrelated targets were obtained. Among them, 80 target proteins were shared proteins, which were the target genes related to the treatment of IBS by SLBZP, as shown in Table 2.

2.3. Target Interaction Network. As shown in the results of the target interaction network in Figure 5, there were 1023 intertarget relationships among 80 targets; on average, each target had potential interactions with 26 targets. The local clustering coefficient was 0.662 , and the protein-protein interaction (PPI) enrichment $p$ value was $<0.001$, suggesting

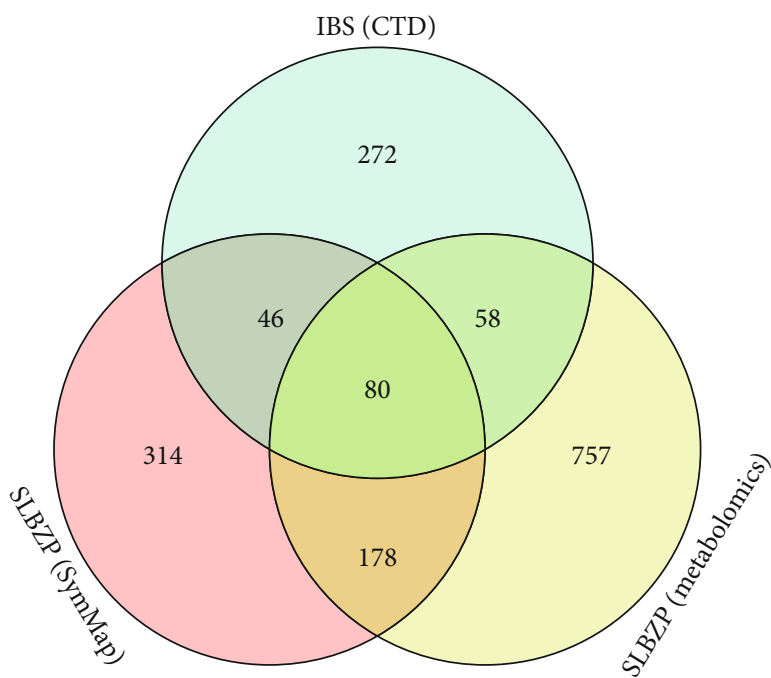

FIGURE 4: Venn diagram of target genes related to IBS treated with SLBZP. 
TABLE 2: SLBZP for the treatment of IBS-related target genes. Bold italics are the core targets.

\begin{tabular}{|c|c|c|c|c|c|c|c|}
\hline CCND1 & CASP3 & MMP9 & ACHE & PGR & HTR3A & XIAP & PLA2G4A \\
\hline IL1B & GAPDH & MPO & ALOX5 & PLA2G2A & NQO1 & GCG & CCR2 \\
\hline JUN & HMOX1 & RELA & BCL2 & PON1 & OPRD1 & APP & NR1H3 \\
\hline NOS2 & ICAM1 & SELE & CTNNB1 & PTGS1 & OPRK1 & CASP7 & GABBR1 \\
\hline$N R 3 C 1$ & IL2 & STAT1 & ESR1 & CYP1A1 & PARP1 & PTGES & RXRA \\
\hline PPARG & MAPK1 & VCAM1 & GSK3B & CYP1A2 & PPARA & GLA & SLC6A2 \\
\hline PTGS2 & МАРКЗ & МАРК 8 & KCNH2 & CYP3A4 & RXRB & MMP13 & SLC6A3 \\
\hline$T N F$ & MMP1 & ELANE & MAOA & DRD2 & SELP & MMP7 & SLC6A4 \\
\hline$V E G F A$ & MMP2 & STAT3 & MAOB & GSR & $\mathrm{TH}$ & RPS6KB1 & $\mathrm{XDH}$ \\
\hline$A K T 1$ & MMP3 & TLR4 & OPRM1 & GUSB & TRPV1 & CYP2D6 & $\mathrm{ABCB} 1$ \\
\hline
\end{tabular}

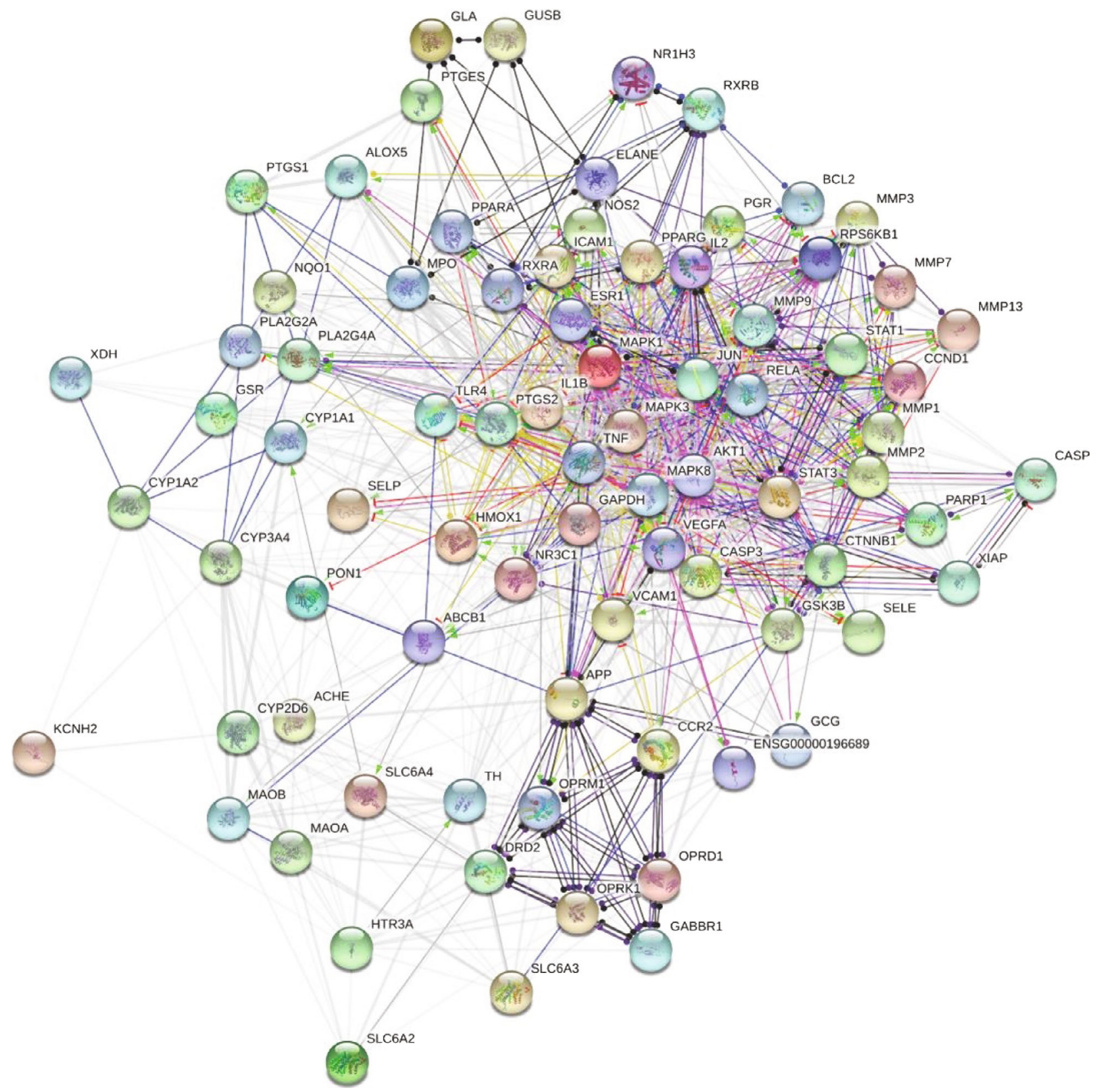

FIGURE 5: Intertarget interaction network of SLBZP in the treatment of IBS. 


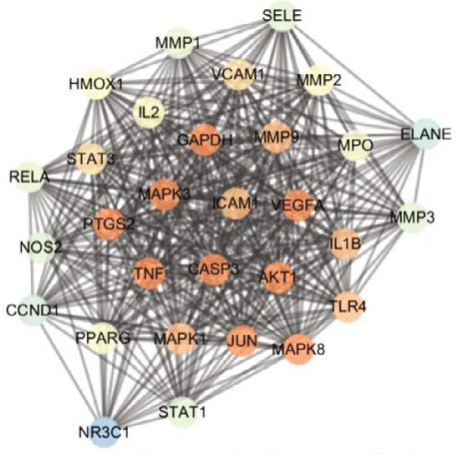

Cluster 1: score $=27.034$

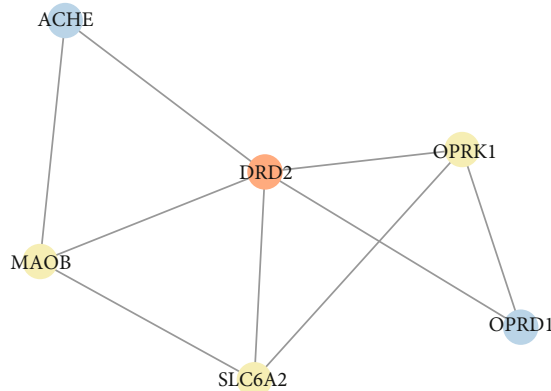

Cluster 4 : score $=3.6$

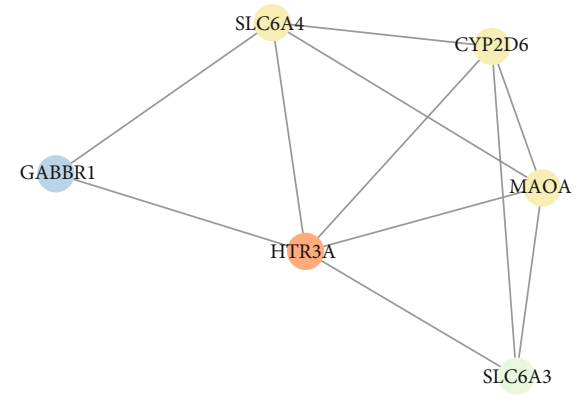

Cluster 2: score $=4.4$

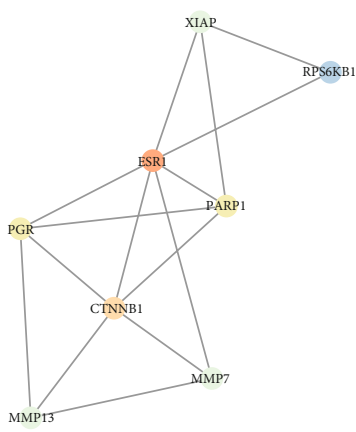

Cluster 3: score $=4.286$

Figure 6: Cluster analysis results of the target interaction network of SLBZP in the treatment of IBS. Note that the warmer the node color, the higher the importance of the node in the network.

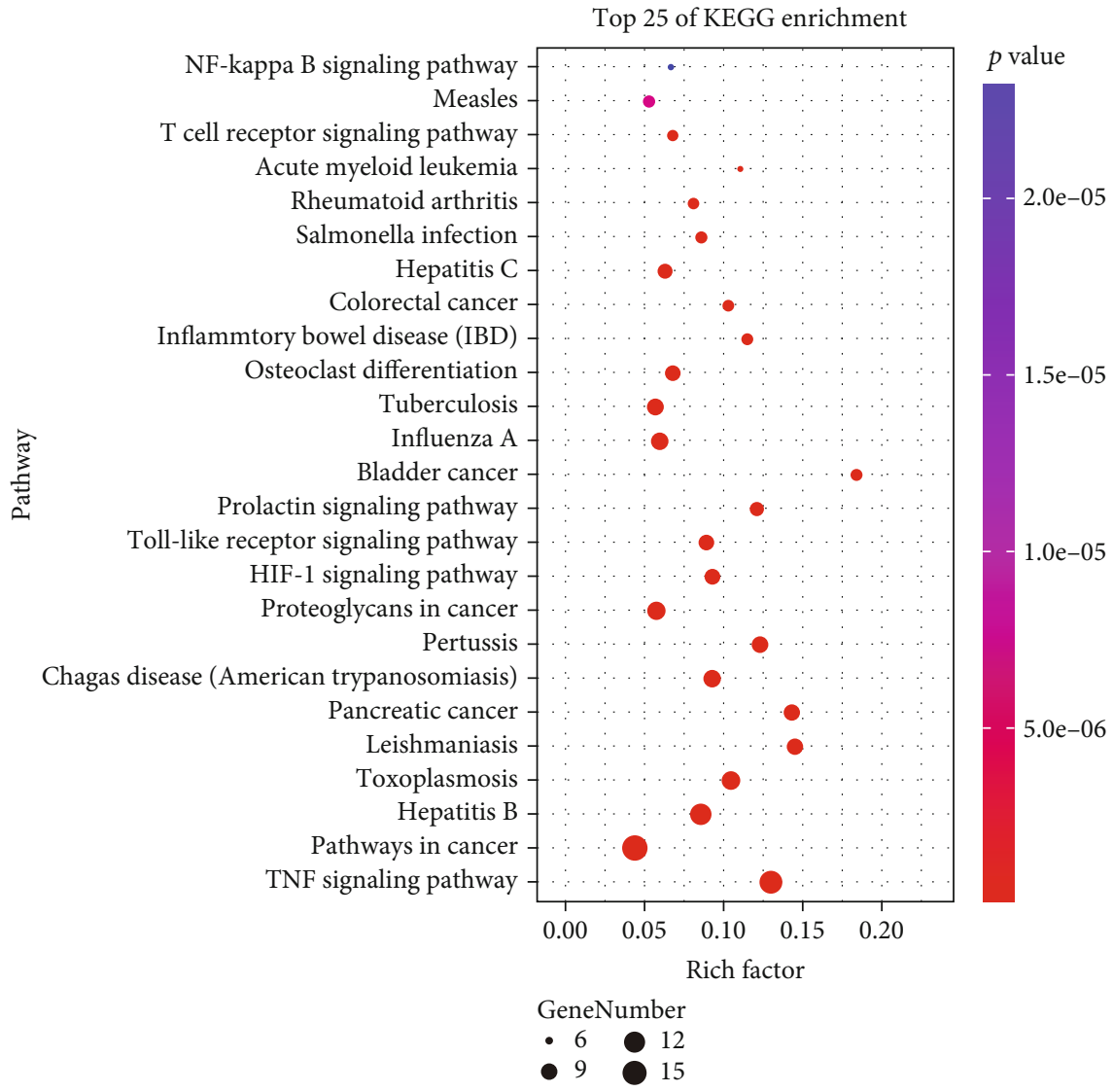

FIGURE 7: Core target pathway enrichment bubble chart of SLBZP in IBS therapy (top 25\%). 


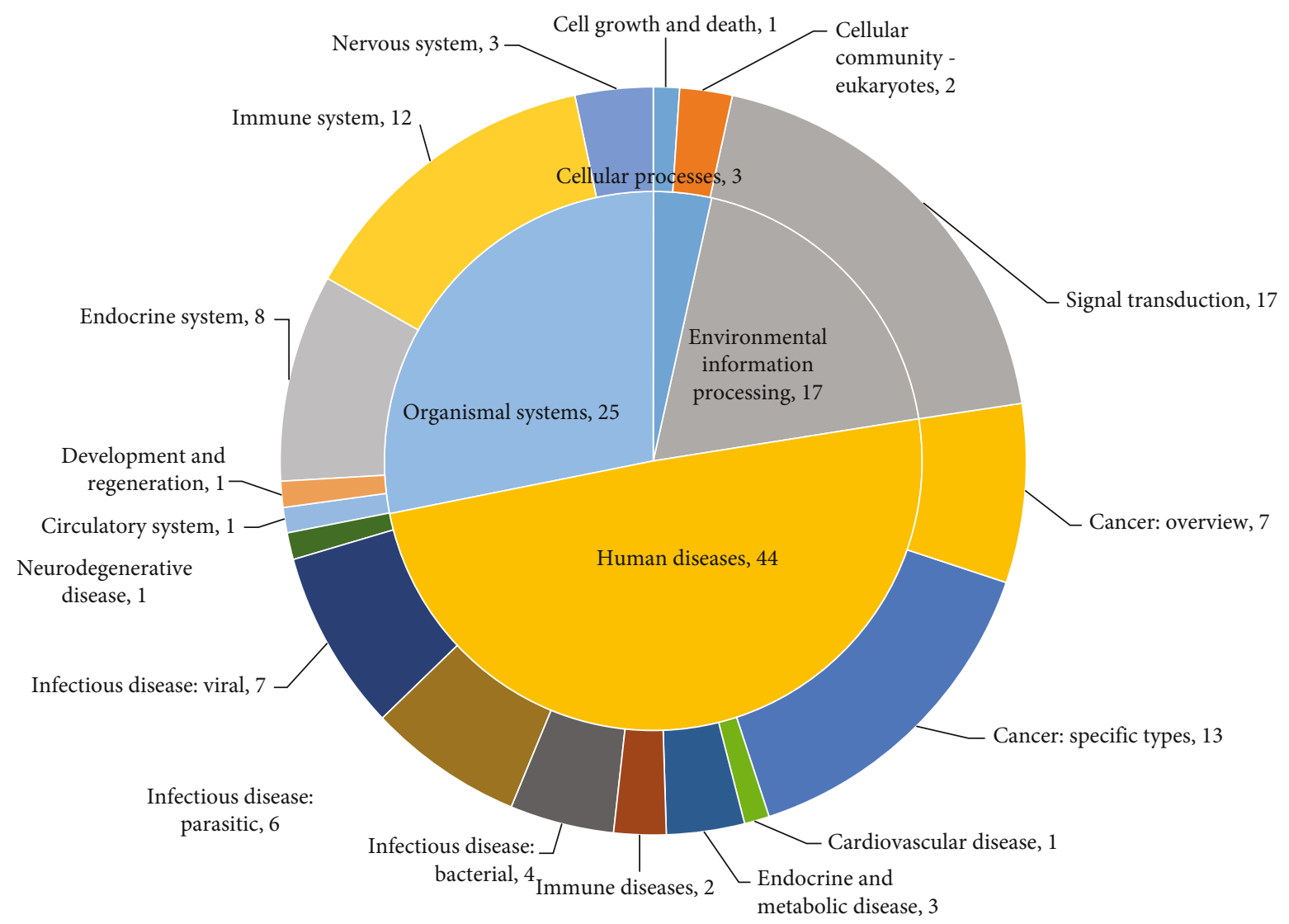

FIGURE 8: Double-loop diagram of enrichment and classification of the core target pathway of SLBZP in IBS therapy.

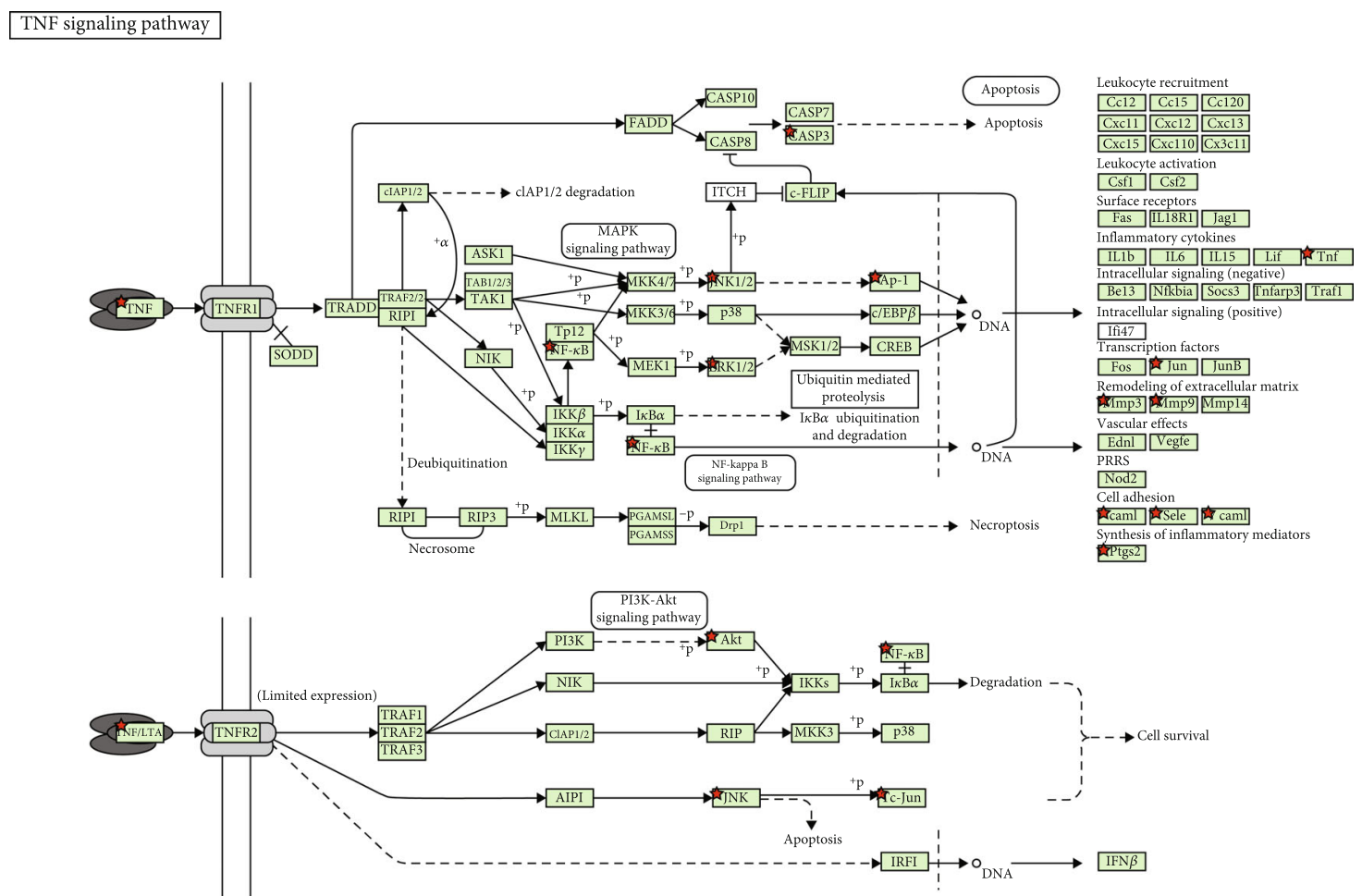

FIGURE 9: Enrichment results of the TNF signaling pathway. Note that the red pentagram is the protein mapped from SLBZP. 


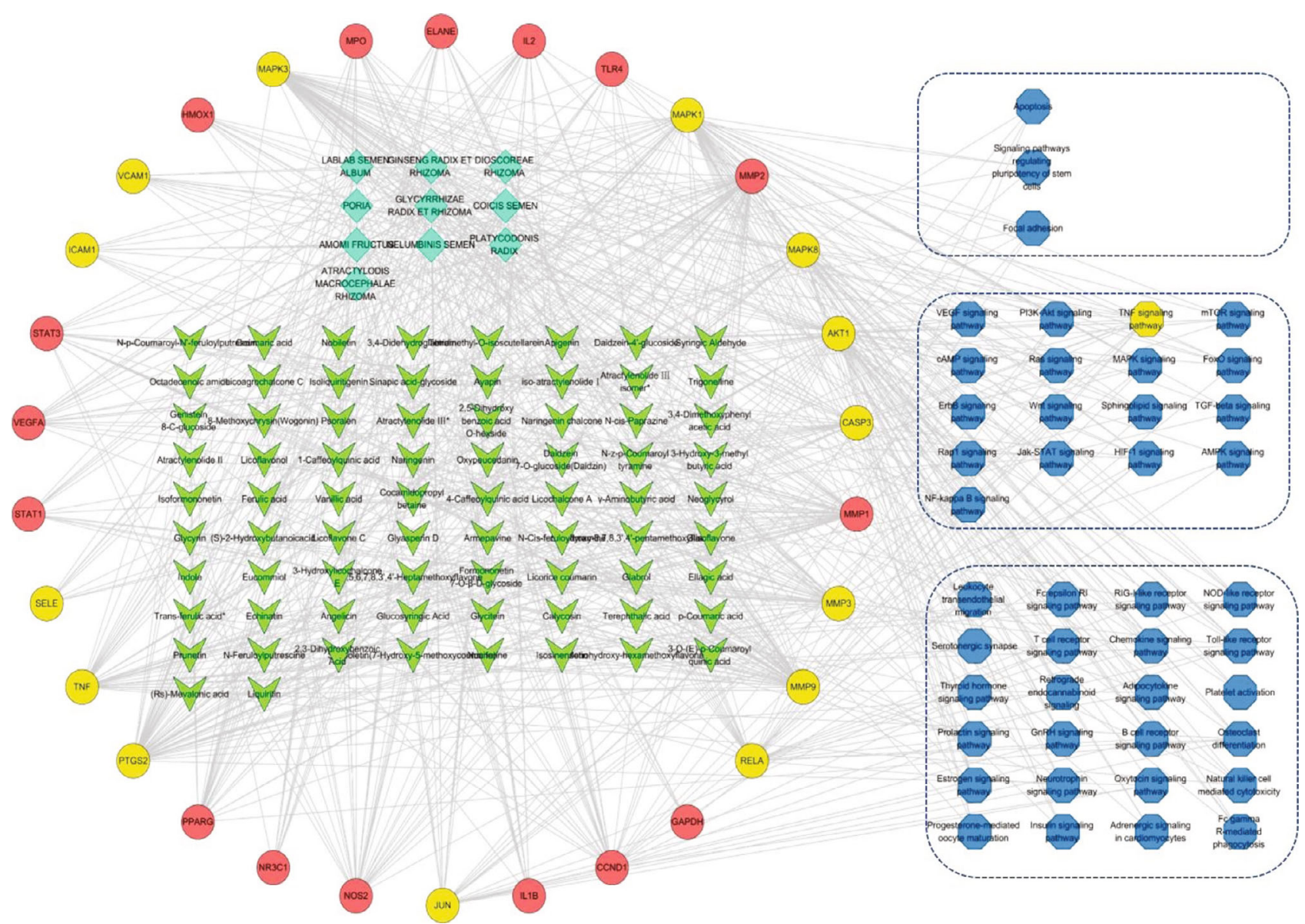

Figure 10: The "drug-molecule-target-pathway" network of SLBZP in the treatment of IBS. Note that the green diamond node is the traditional Chinese medicine, the green arrow node is the molecule, the circular node is the target protein, the hexagonal node is the pathway, and the three parts from top to bottom represent cellular processes, environmental information processing, and organic systems, respectively. The yellow node is the TNF signaling pathway and its related proteins.

that as a cohort, the proteins were biologically connected to some extent. A total of 5 subnetworks were obtained following cluster analysis of this network, as shown in Figure 6. Among them, cluster 1 had the highest cluster score; this subnetwork contained 30 target proteins, as shown in Table 2. These proteins were the core targets of SLBZP in IBS therapy.

2.4. The Results of Pathway Cluster Analysis. As shown in Figures 7-9, a total of 89 pathways were obtained from the enrichment of core target groups, including 4 categories and 17 subcategories in the Kyoto Encyclopedia of Genes and Genomes (KEGG) database. The four first-level classification categories and proportions among them were human diseases (49.4\%), organismal systems (28.1\%), environmental information processing (19.1\%), and cellular processes (3.4\%). The pathway with the highest degree of enrichment was the tumor necrosis factor (TNF) signaling pathway $(p<0.001)$; the most abundant pathway was signal transduction.

2.5. The Results of Pathway Enrichment Analysis. Figures 10 and 11 display the constructed first-level classification path- ways of non-"human diseases" and the network with related targets and Chinese medicines. In the total network nodes, Lablab semen album (degree $=29)$, transferulic acid (degree $=12)$, and TNF signaling (degree $=14$ ) were the most aggregated traditional Chinese medicine, small molecule, and pathway, respectively. In the network nodes of the TNF signaling pathway, the Chinese medicines with the highest aggregation were Lablab semen album and Glycyrrhizae radix et rhizoma (degree $=11$ ), and the small molecules with the highest aggregation were oxypeucedanin and $3,5,6,7,8,3^{\prime}, 4^{\prime}$-heptamethoxyflavone (degree $\left.=4\right)$.

2.6. The Results of Molecular Docking. According to the above results, molecular docking of the TNF protein was carried out; the results are shown in Figure 12. Daidzein 7O-glucoside (daidzin) was the most closely bound to TNF with a binding energy of $-10.3 \mathrm{kcal} / \mathrm{mol}$; it interacted with 11 amino acids by hydrogen bonds.

\section{Discussion}

There are many causes of IBS, but the etiology is still not clear. Studies have shown that IBS may occur following only 


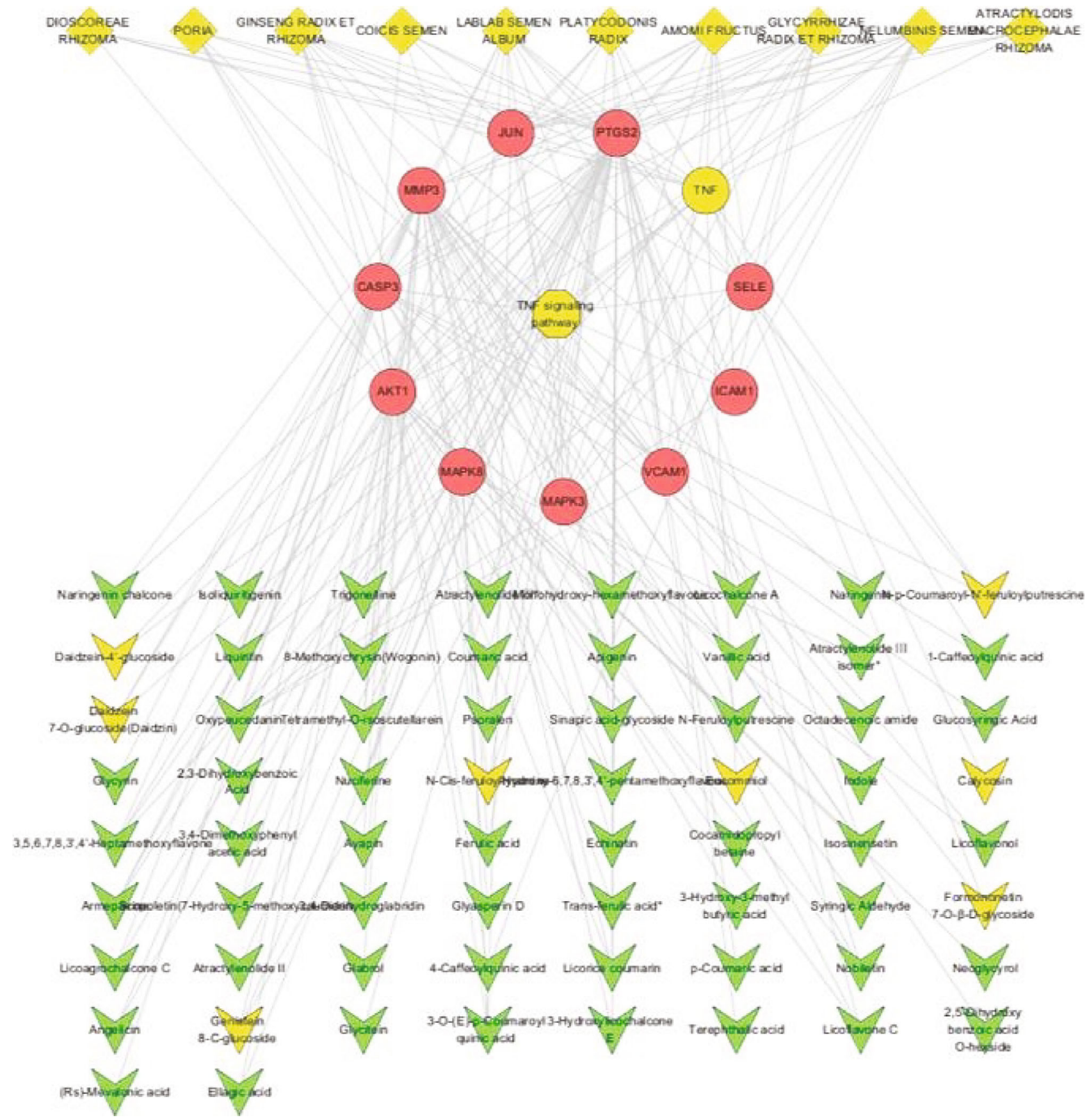

FIgURE 11: The "drug-molecule-target-pathway" network of SLBZP in treating IBS through the TNF signaling pathway. Note that the green diamond node is the traditional Chinese medicine, the green arrow node is the molecule, the circular node is the target protein, and the hexagonal node is the pathway. The yellow nodes are TNF proteins, related small molecules, and traditional Chinese medicine.

slight changes and disturbances, such as the improvement of modern social health conditions or personal hygiene habits or short-term infection or stress [16]. Owing to the complexity of the causes of the disease, there is a wide selection of potential treatments. IBS can seriously affect patients' quality of life and occupational productivity [17]. The total cost of IBS treatment in China has reached 123.83 billion yuan.

In 2018, the American Society of Gastroenterology published a consensus opinion on IBS therapy [3], recommending that in addition to nondrug therapies such as exercise and diet control, secretagogues such as linaclotide and plecanatide and cellulose drugs should be offered for IBS-C. Eluxadoline and alosetron are suggested for IBS-D, but the quality of evidence for this is weak. However, each treatment has its own indications and contraindications, together with inevitable adverse event profiles. For example, linaclotide is generally considered to be a safe and well-tolerated drug, but dose-dependent diarrhea still occurs [18]. In addition, the http://Drugs.com/ database suggests that in nonclinical studies on newborn mice, a single oral dose of linaclotide 


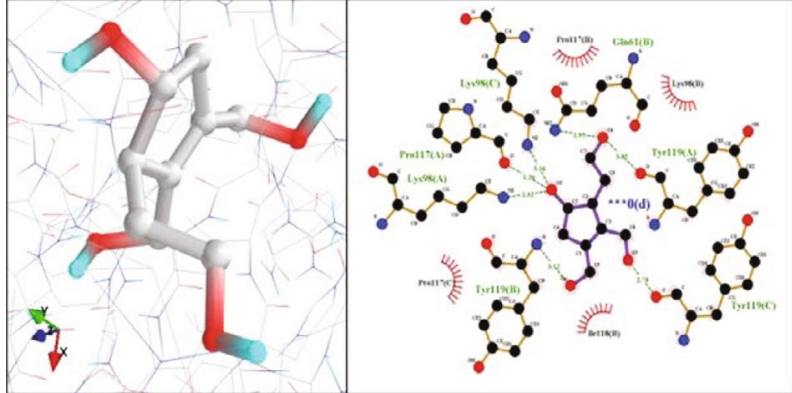

(a)

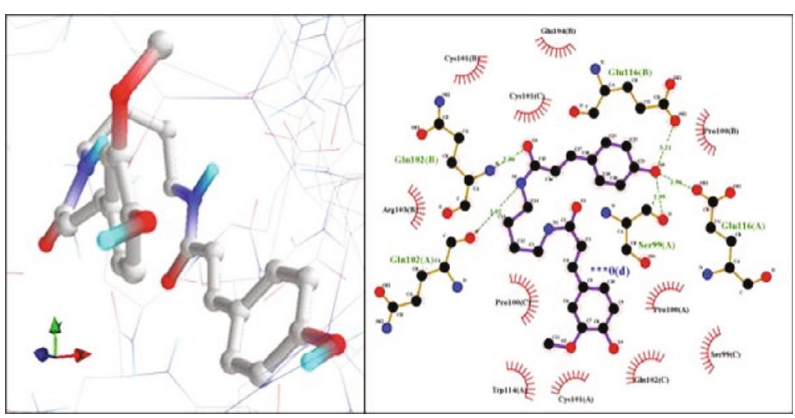

(c)

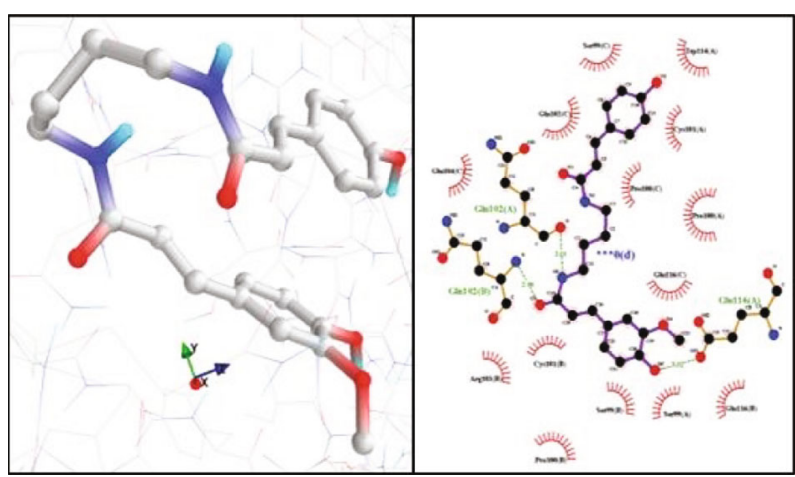

(e)

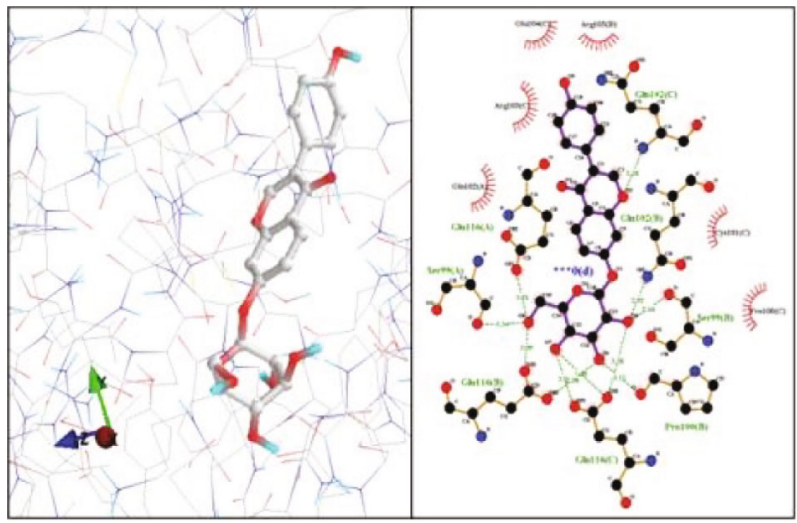

(g)

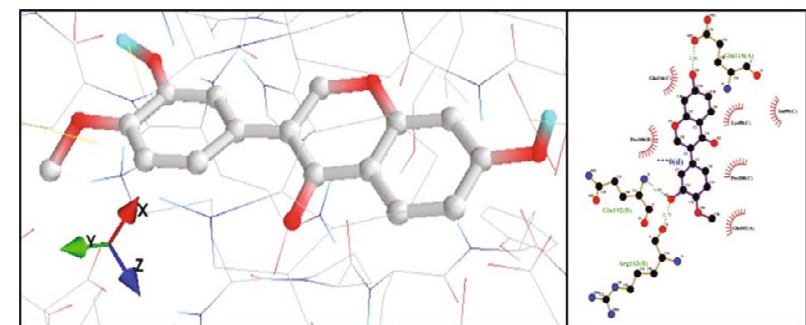

(b)

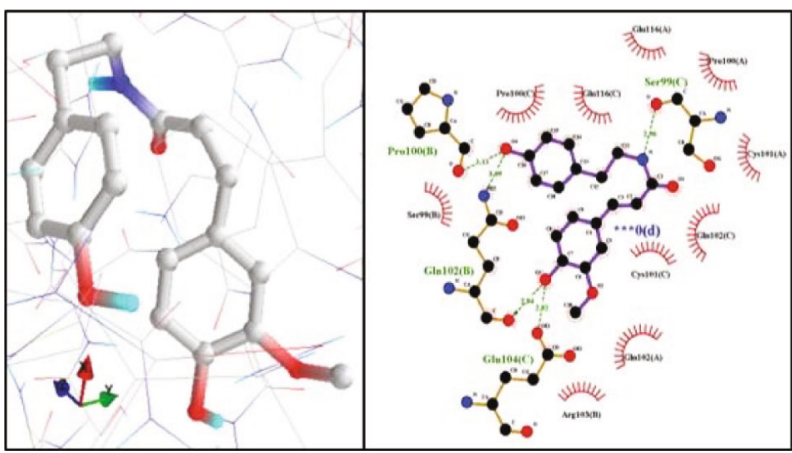

(d)

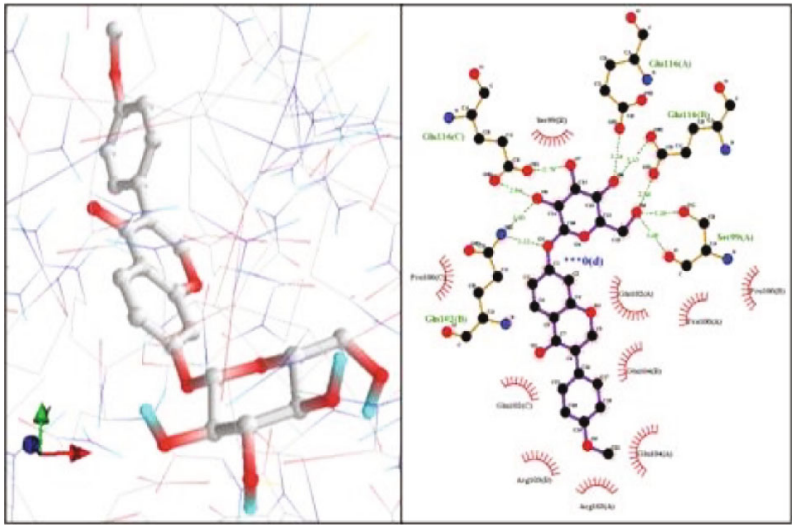

(f)

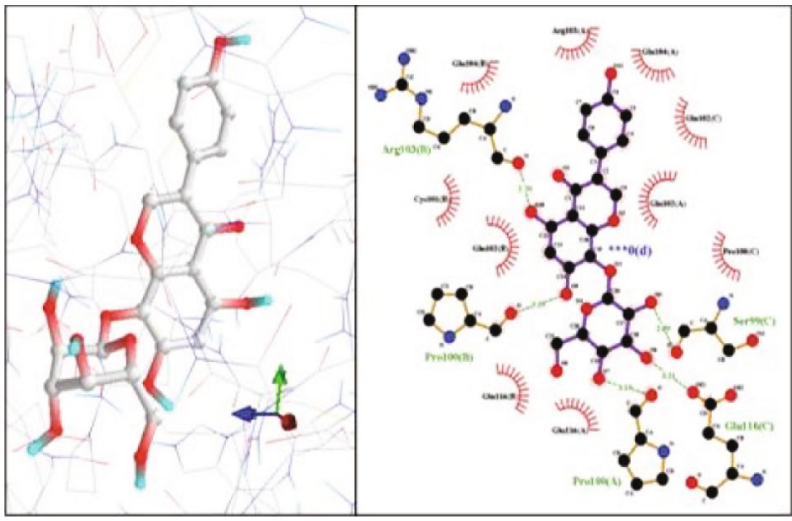

(h)

FIGURE 12: Docking results of TNF-related molecules and their binding energies: (a) eucommiol, $-6.2 \mathrm{kcal} / \mathrm{mol}$; (b) calycosin, $-8.9 \mathrm{kcal} / \mathrm{mol}$; (c) N-p-coumaroyl-N ${ }^{\prime}$-feruloylputrescine, $-7.4 \mathrm{kcal} / \mathrm{mol}$; (d) $49 . \mathrm{N}$-cis-feruloyltyramine, $-8.6 \mathrm{kcal} / \mathrm{mol}$; (e) daidzein- $4^{\prime}$-glucoside, $-8.0 \mathrm{kcal} /$ mol; (f) 79.formononetin 7-O- $\beta$-D-glycoside, -10.4 kcal/mol; (g) daidzein 7-O-glucoside (daidzin), -10.3 kcal/mol; (h) genistein 8-Cglucoside, $-9.4 \mathrm{kcal} / \mathrm{mol}$. 


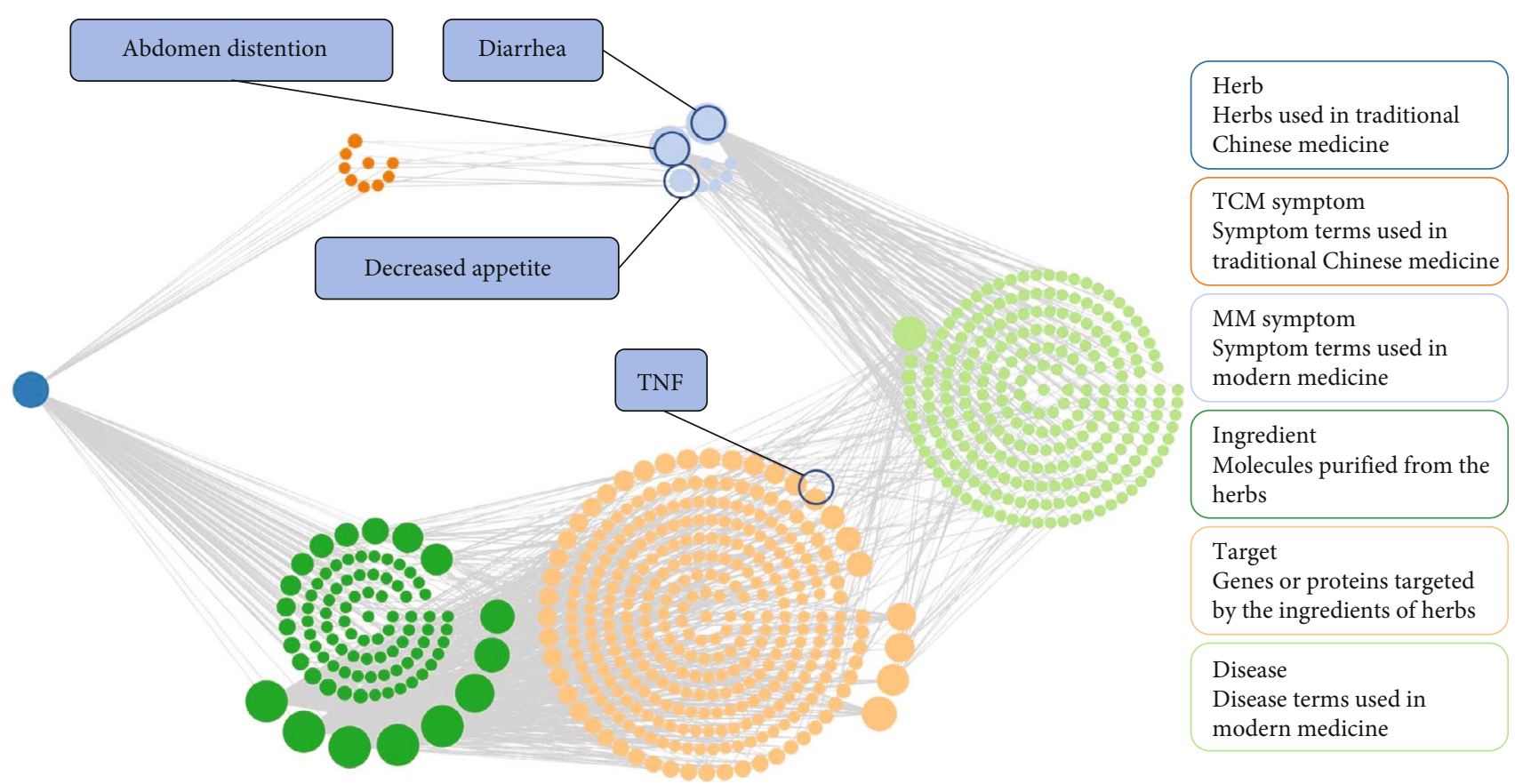

FIGURE 13: Lentil-related "molecule-target-disease-symptom" relationship network. Note that data is from the SymMap database.

can cause dehydration and death, as well as abdominal pain, stomach ache, severe diarrhea, and other common side effects. Eluxadoline increased the risk of pancreatitis. In the United States, adverse event logs relating to eluxadoline, recorded between January and September 2016, revealed that 98 patients with IBS-D had pancreatitis due to taking eluxadoline, resulting in 2 deaths [19]. It is therefore paramount to discover an efficacious, safe, and cost-effective drug which could even be used for the early clinical issues that arise before IBS becomes a major priority.

There are ten kinds of Chinese herbal medicine in SLBZP, among which lentil is the sovereign medicine. Studies have suggested that lentils within this compound are a potential core Chinese workhorse. The traditional Chinese medicine, white lentil, is the dried mature seed of the legume lentil Dolichos lablab L., which was first recorded in the "famous doctors' records" in the late Han Dynasty over 1500 years ago. In the Chinese Pharmacopoeia 2015 Edition, it is documented that this substance can be used as therapy for IBS-related clinical symptomatology, such as loss of appetite, loose stools, and abdominal discomfort, as shown in Figure 13. Among the detected secondary metabolites, most of them have been proven to have certain anti-inflammatory, systemic, and regeneration-promoting effects, of

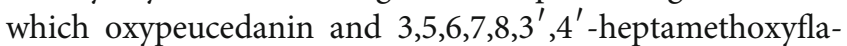
vone were deemed the most enriched. Oxypeucedanin is an open channel blocker, acting on the hKv1.5 channel [20], and can inhibit the growth and cellular life cycle of melanoma cells [21]. Heptamethoxyflavone substances can participate in the regulation of platelet antiadhesion activity [22]. Lentil is the homologous product of medicine and food. It has no toxic side effects on the human body after reasonable cooking, while oxypeucedanin and 3,5,6,7,8,3', $4^{\prime}$-heptamethoxyflavone are small molecular compounds with certain potential pharmacological properties, so they can be used as possible follow-up research and development drugs.

Among the disease pathways suggested by the KEGG database pathway enrichment results, in addition to tuberculosis, pertussis, and other pathology pathways that are not directly related to IBS, there are predominantly two kinds of IBS-related diseases, i.e., inflammatory bowel disease (IBD) and colorectal cancer. IBS and IBD are two completely different pathologies. At present, with respect to the relationship between the two, studies have pointed out that about one-third of patients with IBD have persistent intestinal sensation and movement and intestinal flora abnormalities, which may cause symptoms similar to IBS [23]. In terms of the relationship between IBS and colorectal cancer, some studies have suggested that no association between these disease processes has been found. However, it is clear that there are many cases of misdiagnosis caused by similar symptoms [24]. Combined with the results of this study, in addition to IBS, SLBZP may also be used as a potential candidate drug for IBD and colorectal cancer.

TNF was selected for molecular docking because as an important cytokine, it is able to trigger a variety of intracellular signal pathways and is the initiating factor of the TNF signal pathway. Activated TNF will activate this pathway and induce downstream leukocyte recruitment and inflammatory factor release. It is involved in inducing cellular necrosis and survival, inflammation, and immunity. One of the most significant proinflammatory cytokines, TNF- $\alpha$, is a principal factor in vasodilation and edema and in the epithelial adhesion of leukocytes through adhesion molecule expression. It also governs hemostasis, promotes oxidative stress in inflammatory areas, and indirectly gives rise to pyrexia [25]. At present, studies have suggested that there 
is an imbalance of TNF in patients with IBS [26]. This change may be related to the release of intestinal microbiota and brain neurotransmitters, mediated via the "brain-gutbacteria" axis [27]. Although IBS is not an inflammatory disease per se, it is undeniable that its pathological features have similar inflammatory manifestations. Early inhibition of an inflammatory response through anti-inflammatory agents may therefore be an effective IBS treatment. Such substances include daidzin in SLBZP, which has been shown to offer a certain degree of anti-inflammatory influence through the TNF signaling pathway [28]. Thus, it is concluded that the latter is the mechanism through which SLBZP mainly exhibits an anti-inflammatory effect.

\section{Conclusion}

To summarize, a network, founded on network pharmacology technology, was constructed in order to predict the interaction between substances in SLBZP and target genes. The data demonstrated that SLBZP may be a significant factor in IBS therapy by acting on the TNF signaling pathway. Based on the analysis of systems biology, it was speculated that SLBZP may not only be a player in multiple mechanisms that combine synergistically to treat IBS but also contribute to factors that diminish the IBS incidence. In addition, SLBZP may also be a potential treatment for IBD and colorectal cancer. However, the results obtained by systems biology analysis still need to be verified by pharmacological methods with the aid of omics technology.

\section{Method}

\subsection{Metabolomics Analysis}

5.1.1. Sample Preparation and Extraction. Three sachets, each containing $6 \mathrm{~g}$ of SLBZP (Beijing Tongrentang Pharmaceutical Co., Ltd.), were used for the study (in order to ensure the stability of the results, one bag was used for each measurement, and the measurement was repeated 3 times). The substance was comminuted for 90 seconds in a mixer mill (MM 400, Retsch) with zirconia beads at $30 \mathrm{~Hz}$. Centrifugation was then performed at $10,000 \mathrm{~g}$ for a period of 10 minutes. The extract was absorbed (CNWBOND CarbonGCB SPE Cartridge, $250 \mathrm{mg}, 3 \mathrm{~mL}$; ANPEL, Shanghai, China). Filtration was then performed prior to analysis by liquid chromatography-electrospray ionization-mass spectrometry (LC-ESI-MS).

5.1.2. High-Performance Liquid Chromatography Conditions. An LC-ESI-MS system (high-performance liquid chromatography (HPLC) and Shim-pack ultraperformance liquid chromatography (UFLC), Shimadzu CBM30A system; mass spectrometry (MS), Applied Biosystems 6500 triple quadrupole-linear ion trap (QTRAP)) was used to analyze the resulting extracts. The following analytical conditions were employed: HPLC column: Waters ACQUITY UPLC HSS T3 C18 $(1.8 \mu \mathrm{m}, 2.1 \mathrm{~mm} * 100 \mathrm{~mm})$; solvent system: water ( $0.04 \%$ acetic acid) and acetonitrile (0.04\% acetic acid); and gradient program: 100:0 V/V at $0 \mathrm{~min}, 5: 95 \mathrm{~V} / \mathrm{V}$ at $11.0 \mathrm{~min}, 5: 95 \mathrm{~V} / \mathrm{V}$ at $12.0 \mathrm{~min}, 95: 5 \mathrm{~V} / \mathrm{V}$ at $12.1 \mathrm{~min}$, and
95: $5 \mathrm{~V} / \mathrm{V}$ at $15.0 \mathrm{~min}$. A flow rate of $0.40 \mathrm{~mL} / \mathrm{min}$ was utilized; the temperature was maintained at $40^{\circ} \mathrm{C}$, and $2 \mu \mathrm{L}$ injection volume was used. The effluent was alternately connected to an electrospray ionization-triple quadrupole-linear ion trapmass spectrometry (ESI-QTRAP-MS/MS).

5.1.3. ESI-QTRAP-MS/MS. The linear ion trap (LIT) and triple quadrupole (QQQ) scans were acquired on a QQQ-LIT (QTRAP) mass spectrometer and an API 6500 QTRAP LC/MS/MS system. The system incorporated an electrospray ionization (ESI) turbo ion spray interface, which operated under positive ion mode, and was controlled by Analyst 1.6.3 software (AB Sciex). The following working parameters of the ESI source were deployed: ion source and turbo spray; source temperature $500^{\circ} \mathrm{C}$; ion spray voltage $5500 \mathrm{~V}$; and ion source gas I and gas II and curtain gas at 55, 60, and $25.0 \mathrm{psi}$, respectively; collision gas was high. The instrument was tuned and mass calibrated with $10 \mu \mathrm{mol} / \mathrm{L}$ and $100 \mu \mathrm{mol} / \mathrm{L}$ propylene glycol solutions in QQQ and LIT modes, respectively. The QQQ scans were obtained as multiple reaction monitoring (MRM) experiments; collision gas (nitrogen) was set to 5 psi. The declustering potential and collision energy of a single MRM conversion were further optimized. In accordance with the metabolites eluted during this period, a specific set of MRM transitions was monitored for each timeframe.

5.1.4. Qualitative Analysis of Metabolites. Based on the Malware Database (MetWare) and public databases of metabolite information, respectively, the primary and secondary MS spectrum data were analyzed qualitatively. Iterative isotope signals containing potassium, sodium, and ammonium ions, together with repeating signals of fragment ions of other high-molecular weight compounds, were removed in the process. The structure of metabolites was analyzed with reference to MassBank, KNAPSAcK, the Human Metabolome Database (HMDB) [29], the Metabolome Tomato Database (MoTo DB), and the Metabolite Link (METLIN) [30] as well as to additional existing mass spectrometry public databases.

\subsection{Network Pharmacology Analysis}

5.2.1. Prediction of Potential Targets of SLBZP in the Treatment of IBS. Searches were performed in PubChem and ChemSpider databases to determine the molecular structure of small compounds obtained by metabolomics. The database SwissTargetPrediction was utilized for target fishing in order to obtain related targets for secondary metabolites. The SymMap database was utilized to search for all known target proteins of traditional Chinese medicines within SLBZP [31]; these were merged with the medicine targets. Duplicates were eradicated so as to retain the unique values. A search for IBS genes was conducted using the Comparative Toxicogenomics Database. Retaining the secondary metabolite targets, the known targets in the database and the common gene targets of IBS genes produced the potential targets of SLBZP in IBS treatment.

5.2.2. Constructing a Network of Interaction Relationships between Analytical Targets. The potential targets of the proven prescriptions for the treatment of diarrhea were 
entered into the STRING database, the organization selected was "Homo sapiens," and the function "search" was clicked in order to analyze and obtain the PPI. The PPI network was imported into Cytoscape (v3.7.2) software, and the MCODE plugin was used to cluster the PPI network in order to obtain the core target group for the treatment of diarrhea.

5.2.3. Construction of the Core "Molecule-Target-Pathway" Network. The core target group was entered into the DAVID database in order to perform enrichment analysis and to obtain the potential KEGG pathway. In the acquired pathways and according to the relationship between a target and the corresponding Chinese medicine, Cytoscape (v3.7.2) software was utilized in order to build a "Chinese medicine-molecule-target-pathway" network of nonspecific disease pathways so as to visualize the network relationship.

5.2.4. Molecular Docking Analysis. Molecular docking was used to verify the marker proteins in the most enriched pathway. The 3-dimensional structure of the human target protein was downloaded from the Protein Data Bank (PDB) with the PDB ID of "1TNF" and stored in PDB format. Dehydration and hydrotreatment of the protein were carried out using AutoDockTools (v4.2) software and saved in PDBQT format. Following energy minimization, the 3-dimensional structure of the small molecular compound relating to SLBZP was saved in PDBQT format. The structure of the small molecule and the corresponding target protein was imported into PyRx (v0.9.8) software, and the AutoDock Vina algorithm was employed for molecular docking. According to the docking scoring results, the combination potential between the two was evaluated. The docking results were exported as PDB files; $\mathrm{PDB}$ format files of ligand-receptor binding were generated by PyMOL (v2.4.1) software, and 2-dimensional structure diagrams of ligand-receptor binding were generated by LigPlot (v4.5.3) software.

\section{Abbreviations}

IBS:

SLBZP:

AChE:

ALOX5:

BCL2:

CCND1:

CTNNB1:

TNF:

IBS-C:

IBS-D:

IBS-M:

IBS-U:

PPI:

KEGG:

IBD:

LC-ESI-MS:
HPLC:

UFLC:

MS:

QTRAP:

ESI-QTRAP-MS/

MS:

LIT:

QQQ:

ESI:

MRM:

HMDB:

MoTo DB:

METLIN:

PDB:

\section{Data Availability}

All data are available in the manuscript, and they are shown in figures, tables, and supplementary files.

\section{Consent}

Consent is not applicable.

\section{Conflicts of Interest}

All of the authors report no relationships that could be construed as a conflict of interest.

\section{Authors' Contributions}

The contributions of the authors involved in this study are as follows: ZSS: study concept and design and acquisition of data; MM: analysis and interpretation of data and drafting of the manuscript; BC: acquisition of data, analysis and interpretation of data, and addition of experiment results; WB: material support, analysis and interpretation of data, and critical revision of the manuscript; and ZLQ, LZ, and LDY: revision of the manuscript and study supervision. All authors approved the final version of the manuscript.

\section{Acknowledgments}

The study was funded by the Scientific research "Pei Yu" project of Beijing Municipal Hospital Administration (PZ2018011).

\section{Supplementary Materials}

Please find detailed data in the supplementary file, including the original data of SymMap, metabolomics, and molecular docking of Shenling Baizhu powder. "Shenling Baizhu powder SymMap" includes all herbal targets and combined targets. "Shenling Baizhu powder metabolomics" includes two-dimensional structural data analysis. "Molecular docking" involves molecule-target docking and the results. (Supplementary Materials) 


\section{References}

[1] R. M. Lovell and A. C. Ford, "Global prevalence of and risk factors for irritable bowel syndrome: a meta- analysis," Clinical Gastroenterology and Hepatology, vol. 10, no. 7, pp. 712721.e4, 2012.

[2] W. D. Chey, J. Kurlander, and S. Eswaran, "Irritable bowel syndrome: a clinical review," Journal of the American Medical Association, vol. 313, no. 9, pp. 949-958, 2015.

[3] A. C. Ford, P. Moayyedi, W. D. Chey et al., "American College of Gastroenterology monograph on management of irritable bowel syndrome," American Journal of Gastroenterology, vol. 113, pp. 1-18, 2018.

[4] A. C. Ford, P. Moayyedi, B. E. Lacy et al., "American College of Gastroenterology monograph on the management of irritable bowel syndrome and chronic idiopathic constipation," American Journal of Gastroenterology, vol. 109, pp. S2-S26, 2014.

[5] L. Chao, Z. Li, J. Zhou et al., "Shen-ling-bai-zhu-san improves dextran sodium sulfate-induced colitis by inhibiting caspase-1/ caspase-11-mediated pyroptosis," Frontiers in Pharmacology, vol. 11, 2020.

[6] H. J. Ji, N. Kang, T. Chen et al., "Shen-ling-bai-zhu-san, a spleen-tonifying Chinese herbal formula, alleviates lactoseinduced chronic diarrhea in rats," Journal of Ethnopharmacology, vol. 231, pp. 355-362, 2019.

[7] S. Zhang, L. Lin, W. Liu et al., "Shen-Ling-Bai-Zhu-San alleviates functional dyspepsia in rats and modulates the composition of the gut microbiota," Nutrition Research, vol. 71, pp. 89-99, 2019.

[8] W. J. Lv, C. Liu, Y. F. Li et al., "Systems pharmacology and microbiome dissection of Shen Ling Bai Zhu San reveal multiscale treatment strategy for IBD," Oxidative Medicine and Cell Longevity, vol. 2019, article 8194804, pp. 1-30, 2019.

[9] K. Shi, L. Qu, X. Lin et al., "Deep-fried Atractylodis Rhizoma protects against spleen deficiency-induced diarrhea through regulating intestinal inflammatory response and gut microbiota," International Journal of Molecular Science, vol. 21, no. 1, p. 124, 2020.

[10] W. Lv, C. Liu, C. Ye et al., "Structural modulation of gut microbiota during alleviation of antibiotic- associated diarrhea with herbal formula," International Journal of Biological Macromolecules, vol. 105, Part 3, pp. 1622-1629, 2017.

[11] M. J. Son, Y. E. Kim, Y. I. Song, and Y. H. Kim, "Herbal medicines for treating acute otitis media: a systematic review of randomised controlled trials," Complementary Therapies in Medicine, vol. 35, pp. 133-139, 2017.

[12] Q. H. Yang, Y. J. Xu, Y. Z. Liu et al., "Effects of ChaihuShugan-San and Shen-Ling-Bai-Zhu-San on p38 MAPK pathway in Kupffer cells of nonalcoholic steatohepatitis," EvidenceBased Complementary and Alternative Medicine, vol. 2014, 8 pages, 2014.

[13] T. H. Chao, P. K. Fu, C. H. Chang, S. N. Chang, F. C. Mao, and C. H. Lin, "Prescription patterns of Chinese herbal products for post-surgery colon cancer patients in Taiwan," Journal of Ethnopharmacology, vol. 155, no. 1, pp. 702-708, 2014.

[14] Y. Wang, P. S. Lee, Y. F. Chen, C. T. Ho, and M. H. Pan, "Suppression of adipogenesis by 5-hydroxy-3,6,7,8,3',4'-hexamethoxyflavone from orange peel in 3T3-L1 cells," Journal of Medicinal Food, vol. 19, no. 9, pp. 830-835, 2016.

[15] A. L. Hopkins, "Network pharmacology: the next paradigm in drug discovery," Nature Chemical Biology, vol. 4, no. 11, pp. 682-690, 2008.
[16] X. Qin, "Damage of the mucus layer: the possible shared critical common cause for both inflammatory bowel disease (IBD) and irritable bowel syndrome (IBS)," Inflammatory Bowel Disease, vol. 23, no. 2, pp. E11-E12, 2017.

[17] N. Sugawara, K. Sato, I. Takahashi et al., "Irritable bowel syndrome and quality of life in a community-dwelling population in Japan," International Journal of Psychiatry in Medicine, vol. 53, no. 3, pp. 159-170, 2018.

[18] S. Mozaffari, S. Nikfar, and M. Abdollahi, "The safety of novel drugs used to treat irritable bowel syndrome," Expert Opinion on Drug Safety, vol. 13, no. 5, pp. 625-638, 2014.

[19] A. J. Gawron and K. Bielefeldt, "Risk of pancreatitis following treatment of irritable bowel syndrome with eluxadoline," Clinical Gastroenterology and Hepatology, vol. 16, no. 3, pp. 378384.e2, 2018.

[20] J. S. Eun, J. A. Park, B. H. Choi, S. K. Cho, D. K. Kim, and Y. G. Kwak, "Effects of oxypeucedanin on hKv1.5 and action potential duration," Biological and Pharmaceutical Bulletin, vol. 28, no. 4, pp. 657-660, 2005.

[21] Y. Kimura, M. Sumiyoshi, M. Sakanaka, M. Taniguchi, and K. Baba, "In vitro and in vivo antiproliferative effect of a combination of ultraviolet-A and alkoxy furocoumarins isolated from Umbelliferae medicinal plants, in melanoma cells," Photochemistry and Photobiology, vol. 89, no. 5, pp. 1216-1225, 2013.

[22] Y. Wang, S. Zhang, Q. Zhou, M. Meng, and W. Chen, "Efficacy of Shenling Baizhu formula on irritable bowel syndrome: a systematic review," Journal of Traditional Chinese Medicine, vol. 40, no. 6, pp. 897-907, 2020.

[23] R. Spiller and G. Major, "IBS and IBD - separate entities or on a spectrum?," Nature Reviews Gastroenterology and Hepatology., vol. 13, no. 10, pp. 613-621, 2016.

[24] C. W. Hsiao, W. Y. Huang, T. W. Ke et al., "Association between irritable bowel syndrome and colorectal cancer: a nationwide population-based study," European Journal of Internal Medicine, vol. 25, no. 1, pp. 82-86, 2014.

[25] H. Zelova and J. Hosek, "TNF- $\alpha$ signalling and inflammation: interactions between old acquaintances," Inflammation Research, vol. 62, no. 7, pp. 641-651, 2013.

[26] M. Bashashati, N. Rezaei, A. Shafieyoun et al., "Cytokine imbalance in irritable bowel syndrome: a systematic review and meta-analysis," Neurogastroenterology and Motility, vol. 26, no. 7, pp. 1036-1048, 2014.

[27] M. Arzani, S. R. Jahromi, Z. Ghorbani et al., "Gut-brain axis and migraine headache: a comprehensive review," The Journal of Headache and Pain, vol. 21, no. 1, p. 15, 2020.

[28] F. Xiao, H. Cui, and X. Zhong, "Beneficial effect of daidzin in dry eye rat model through the suppression of inflammation and oxidative stress in the cornea," Saudi Journal of Biological Science, vol. 25, no. 4, pp. 832-837, 2018.

[29] D. S. Wishart, T. Jewison, A. C. Guo et al., "HMDB 3.0-the Human Metabolome Database in 2013," Nucleic Acids Research, vol. 41, no. D1, pp. D801-D8D7, 2013.

[30] Z. J. Zhu, A. W. Schultz, J. Wang et al., "Liquid chromatography quadrupole time-of-flight mass spectrometry characterization of metabolites guided by the METLIN database," Nature Protocols, vol. 8, no. 3, pp. 451-460, 2013.

[31] Y. Wu, F. Zhang, K. Yang et al., "SymMap: an integrative database of traditional Chinese medicine enhanced by symptom mapping," Nucleic Acids Research, vol. 47, no. D1, pp. D1110-D1117, 2019. 